\title{
Author Correction: Polarons in materials
}

Cesare Franchini([), Michele Reticcioli(i), Martin Setvin and Ulrike Diebold (1)

Correction to: Nature Reviews Materials https://doi.org/10.1038/s41578-021-00289-w, published online 19 March 2021.

The originally published article listed the wrong year for Ref.154 in the $21^{\text {st }}$ row of Table 1: Ref. 154 was published in 2010, not in 2000 . The year has been corrected both in the PDF and in the HTML versions of the article.

https://doi.org/10.1038/s41578-021-00344-6 I Published online 23 June 2021

๑) Springer Nature Limited 2021 